\title{
Disorder-enhanced superconductivity in a quasi-one-dimensional strongly correlated system
}

\author{
A. Lowe $\odot,{ }^{1, *}$ V. Kagalovsky $\odot,^{2}$ and I. V. Yurkevich $\odot^{1}$ \\ ${ }^{1}$ Aston University, School of Informatics \& Digital Engineering, Birmingham B4 7ET, United Kingdom \\ ${ }^{2}$ Shamoon College of Engineering, Bialik/Basel St., Beer-Sheva 84100, Israel
}

(Received 6 January 2021; revised 20 March 2021; accepted 10 May 2021; published 16 July 2021)

\begin{abstract}
We perform an analytical and numerical study of a superconducting instability in quasi-one-dimensional (quasi-1D) disordered systems. Modeling them as an array of Luttinger liquids with Josephson-type interchain coupling, we employed renormalization-group analysis with an extensive search for parameters that support superconductivity enhancement. We have found that this phenomenon is possible in the parameters range that support a latent disorder-driven phase transition between charge- and spin-density-wave phases. Our results may explain the experimental observation of disorder-enhanced superconductivity.
\end{abstract}

DOI: 10.1103/PhysRevResearch.3.033059

\section{INTRODUCTION}

Our understanding of dirty superconductors has been evolving for a few decades now. The original description was based on the Anderson theorem [1] which stated that superconductivity is insensitive to perturbations that do not destroy time-reversal invariance, and, hence do not break Cooper pairs built from exact eigenfunctions which are time-reversal partners of each other. Later it was realized that the assumption of a homogeneous mean-field pairing function breaks down as the system approaches the Anderson metal-insulator transition [2]. These mesoscopic fluctuations of the pairing function eventually destroy superconducting order when the number of attractive electrons in the localization volume falls below one [3]. This superconductor-insulator transition in uniformly (not granulated) disordered systems should occur below the Anderson mobility edge only if the Coulomb interaction can be neglected.

A mechanism for the suppression of superconductivity by Coulomb repulsion was developed by Finkelstein [4]. The Finkelstein effect becomes important for very thin, strongly but homogeneously disordered films, as well as quasi-1D diffusive wires [5]. The essence of the Finkelstein effect is that the Coulomb repulsion between the electrons gets enhanced due to the slow diffusion of electrons in a highly disordered film, which results in the negative contribution to the effective Cooper attraction amplitude at small energy transfer. A similar conclusion has been reached within the nonlinear $\sigma$-model with a nonperturbative saddle-point approximation [6].

Recent research on the interplay between superconducting pairing and disorder led to the observation that if the Coulomb repulsion can be neglected, a new critical superconducting phase close to the mobility edge can be identified. This critical

\footnotetext{
*lowea3@aston.ac.uk

Published by the American Physical Society under the terms of the Creative Commons Attribution 4.0 International license. Further distribution of this work must maintain attribution to the author(s) and the published article's title, journal citation, and DOI.
}

superconducting phase is characterized by the multifractality of the eigenfunctions and, as the result, by the enhancement of the transition temperature with respect to clean $s$-wave superconductivity [7].

The influence of disorder on the temperature of the superconducting transition $T_{c}$ within the nonlinear $\sigma$-model with renormalization-group analysis in $2 \mathrm{D}$ proved that there is a range of interaction parameters where the superconducting temperature can be enhanced even under the inclusion of a short-ranged Coulomb interaction [8,9]. It was shown that in some regime the Anderson localization leads to a strong enhancement of $T_{c}$ due to to the multifractality of wave functions.

The most recent experimental result on the enhancement of superconductivity by disorder was performed on quasi-1D materials [10]. There was a particular signature within the experiment which provided a unique insight into the system: the superconducting transition temperature appeared to be weakly dependent on the disorder strength at weak and strong disorders demonstrating a sharp increase in between, as the disorder increased. This behavior suggests that the system undergoes another phase transition induced by disorder and superconductivity develops at different critical temperatures in those phases. If the phase developing at a stronger disorder is more favourable to the superconducting instability, the dependence of a critical superconducting temperature should acquire a kink-like increase with increase of disorder. It is known [11] that the charge-density-wave (CDW) phase is more amicable to the pairing instability compared to the spindensity-wave (SDW) phase because the CDW phase can be visualized as a spatially modulated distribution of singlets playing the role of pre-existing pairs. If an increase in disorder strength could lead to a transition from SDW to CDW, this would be a reasonable model to describe the superconducting temperature enhancement in quasi-1D systems. In the paper, we show that such a regime indeed exists and the critical temperature experiences a sharp jump in $T_{c}$ with the increase of disorder around the boundary separating SDW and CDW regimes.

There are a few other features of resistivity $\rho$ observed in the experiment: 1) linear in $T$ resistivity over a wide interval of 
rather high temperatures $T, 2$ ) minimum of resistivity at low temperatures $\left.T_{\min }, 3\right)$ steep upturn of $\rho(T)$ at $T<T_{\min }$, and 4) peak of resistivity at $T=T_{\text {peak }}$ followed by sharp drop to zero at $T_{c}$. The most important feature is that all characteristic temperatures experience similar kinklike changes around some critical disorder common for them all. This fact is just another indication of an underlying phase transition defining the rest. Although our research was inspired by the observations [10], we are unable to quantitatively describe all the mentioned features within the standard perturbative renormalization-group (RG) analysis but we will show that all those tendencies are present in the perturbative RG flows implying that our model is adequately reflecting the experiments on quasi-1D materials demonstrating superconductivity.

\section{RESULTS}

\section{A. Model}

At high temperatures $T$, quasi-1D materials do not show dimensional crossover to 3D behavior and can be modelled as an array of $1 \mathrm{D}$ chains without hybridization. The Hamiltonian is thus the sum of Luttinger liquid Hamiltonians [11] describing individual chains with charge $(\eta=\rho)$ and spin $(\eta=\sigma)$ degrees of freedom in each chain and the interchain coupling. The Hamiltonian density $H_{0}$, of uncoupled chains,

$$
\begin{aligned}
H_{0}= & \sum_{i, \eta} \frac{u_{\eta}}{2 \pi}\left[\frac{1}{K_{\eta}}\left(\partial_{x} \phi_{i \eta}\right)^{2}+K_{\eta}\left(\partial_{x} \theta_{i \eta}\right)^{2}\right] \\
& +\frac{y u_{\sigma}}{2 \pi \alpha^{2}} \cos \left(\sqrt{8} \phi_{i \sigma}\right) \\
& +\xi(x) e^{i \sqrt{2} \phi_{\rho}} \cos \sqrt{2} \phi_{\sigma}+\text { c.c. }
\end{aligned}
$$

depends on speed, $u_{\eta}$, and Luttinger parameters, $K_{\eta}$, of the corresponding modes. The parameter $\alpha$ is an ultra-violet cutoff. The dimensionless parameter $y$ is the strength of interaction between electrons with opposite spin. Disorderinduced backscattering is represented by the last term with a white-noise random variable $\xi(x),\left\langle\xi(x) \bar{\xi}\left(x^{\prime}\right)\right\rangle=(2 \pi \alpha)^{2} D_{b}$ $\delta\left(x-x^{\prime}\right)$.

We did not include single-particle hybridization between chains because we assume that the single-particle hybridization is weak and irrelevant in the following sense. The RG equations derived below show that each chain treated individually flows to either a spin-gapped phase (SDW if $y \rightarrow 1$ or CDW if $y \rightarrow-1$, whatever happens first) or to an insulator $(D \rightarrow 1)$. In the former case, the single-particle tunneling is blocked [11] at some temperature $T_{y}$ where $y=1$ (SDW) or $y=-1(\mathrm{CDW})$. At a later point, we stop the RG at a corresponding scale (temperature $T_{d}$ ) and announce the phase insulating without further investigation. Even if the singleparticle hybridization is relevant at low temperatures, this crossover temperature $T_{h y b}$ will be a power-law function of the hybridization strength and we may assume that $T_{h y b} \ll$ $\min \left(T_{y}, T_{d}\right)$.

Anticipating superconductivity to occur in a spin-gapped phase, we include the Josephson coupling term describing the tunneling of singlet pairs between neighboring chains [11]:

$$
H_{J}=-J \sum_{\langle i, j\rangle} \cos \left[\sqrt{2}\left(\theta_{\rho}^{i j}+\phi_{\sigma}^{i j}\right)\right],
$$

where $\theta_{\rho}^{i j}=\theta_{\rho}^{i}-\theta_{\rho}^{j}$ and $\phi_{\sigma}^{i j}=\phi_{\sigma}^{i}-\phi_{\sigma}^{j}$. Although superconductivity will occur in spin-gapped phases where $\phi_{\sigma}^{i j}=0$, the renormalization of the effective Josephson coupling $J$ takes place even before the system developed a gap. That is why we are using full version of it that includes spin degrees of freedom.

The total Hamiltonian $H=H_{0}+H_{J}$ will further be treated within a perturbative two-step RG scheme described in the next section.

\section{B. Two-step renormalization group equations}

The renormalization group equations for the modified by disorder parameters of the Hamiltonian $H_{0}$ can be found in the textbook [11]. In the Appendix, we present those equations and then redefine parameters to introduce the true parameters that do not contain an artificial admixture of the disorder (see Refs. [12,13] for detailed discussion). The equations derived after those redefinitions do not generate spurious inelastic interactions caused by disorder. Introducing a scaling parameter $l=-\ln \left(\Lambda / \Lambda_{0}\right)$ where $\Lambda$ and $\Lambda_{0}$ are running and ultra-violet cutoff energies, we arrive at the following set of RG equations:

$$
\begin{aligned}
\frac{d K_{\rho}}{d l}= & -\frac{1}{4}\left(K_{\rho}^{2}+1\right)\left(K_{\rho}+K_{\sigma}+y-3\right) D \gamma \\
& -\frac{1}{2} \frac{K_{\rho}^{3}}{K_{\rho}^{(0)} u} D, \\
\frac{d K_{\sigma}}{d l}= & -\left[\frac{1}{4}\left(K_{\sigma}^{2}+1\right)\left(K_{\rho}+K_{\sigma}+y-3\right)\right. \\
& \left.-y K_{\sigma}^{2}\right] D \gamma-\frac{1}{2} K_{\sigma}^{2} D-\frac{1}{2} K_{\sigma}^{2} y^{2}, \\
\frac{d y}{d l}= & {\left[1+K_{\sigma}-K_{\rho}+\frac{y}{2}\left(K_{\sigma}^{2}-1\right)\right] D \gamma-D } \\
& +2\left(1-K_{\sigma}\right) y, \\
\frac{d D}{d l}= & -\left(K_{\rho}+K_{\sigma}+y-3\right) D, \\
\frac{d u}{d l}= & \left(\frac{K_{\sigma}^{2}-1}{4 K_{\sigma}}\left(K_{\sigma}+K_{\rho}+y-3\right) \gamma-\frac{K_{\sigma}}{2}\right) u D,
\end{aligned}
$$

where the following notations have been used:

$$
u=\frac{u_{\sigma}}{u_{\rho}^{(0)}}, \quad D=\frac{2 \pi D_{b} \alpha}{\pi u_{\sigma}^{2}}\left(\frac{u_{\sigma}}{u_{\rho}}\right)^{K_{\rho}}, \quad \gamma=\left(\frac{K_{\rho}}{K_{\rho}^{(0)} u}\right)^{K_{\rho}} .
$$

It is well expected that superconductivity emerges in a spingapped phase [14] when either a CDW or a SDW order is dominant. We assume that the plateaus in the experimental graph for the resistivity which reaches a maximum at $T_{\text {peak }}$ immediately followed by the superconducting transition, $T_{\text {peak }} \approx$ $T_{c}$, could be due to a different mechanism controlling the superconducting state. In the context of the RG equations in this paper, the two-step RG scheme should be implemented. When a spin-gapped state emerges, the spin degrees of freedom are frozen, resulting in a new set of spin-gapped RG equations:

$$
\frac{d K_{\rho}}{d l}=-\frac{1}{4}\left(K_{\rho}^{3}-K_{\rho}^{2}+K_{\rho}-3\right) D
$$




$$
\frac{d D}{d l}=-\left(K_{\rho}-3\right) D
$$

The set of initial conditions for these equations are defined by the continuity condition. The exception is the crossover to a SDW phase where the disorder parameter $D$ drops down by a small parameter $\left\langle\cos \sqrt{2} \phi_{\sigma}\right\rangle \propto T_{y}^{K_{\sigma}} \ll 1$. Here $T_{y}$ is the temperature of transition to a SDW phase, which is denoted by $T_{\mathrm{SDW}}$, where this temperature is normalized by the upper cutoff energy such that $T_{y}=T_{\mathrm{SDW}} / \Lambda_{0}$. In what follows, all energies will be normalized by the upper cutoff to make them dimensionless.

The Josephson coupling in a spin-gapped phase is renormalized by the charge degrees of freedom only:

$$
J(l)=J\left(l_{y}\right) \exp \left\{\int_{l_{s}}^{l} d l^{\prime}\left[2-K_{\rho}^{-1}\left(l^{\prime}\right)\right]\right\} .
$$

The dimensional critical temperature $T_{c}=e^{-l_{c}}$ is defined by the condition $J\left(T_{c}\right)=1$.

It is necessary to mention that there are other interchain terms generated by the RG procedure when hybridization between different chains is taken into account. They have a small bare amplitude which is quadratic in the weak hybridization limit but may become relevant. The main effect of the firststep RG procedure is the flowing into either a conducting spin-gapped phase or an insulating state. We are interested in the former case because superconductivity may emerge only in this situation. Choosing a set of corresponding bare values for all of the (fine-tuned) material parameters as the initial conditions for the RG equations guarantee this result. Other weaker (quadratic in hybridization) interchain terms generated by the RG will have some dynamics but their feedback may be neglected at this stage. Entering the spin-gapped phase, multiple interchain terms become irrelevant because they contain either $\phi_{\sigma}$ which is frozen or conjugate to it $\theta_{\sigma}$ which is "delocalized" and smears the corresponding term to zero. The only interchain terms that evolve after entering the spin-gapped phase and must be accounted for in the secondstep RG procedure are those containing the charge degrees of freedom, $\phi_{\rho}$ or $\theta_{\rho}$. Since these variables are mutually conjugate, their scaling dimensions are inversely proportional to each other. The scaling dimension of the Josephson coupling containing what may be called a superconducting phase in the $i$ th chain, $\theta_{\rho, i}$, is equal to $K_{\rho}^{-1}$ and it defines the dynamics of the Josephson coupling in equation (10). The scaling dimension of the interchain CDW terms containing the density field $\phi_{\rho, i}$ is equal to $K_{\rho}$. Aiming at the situation where the system becomes superconducting, we restrict our analysis to the bare values $K_{\rho}^{(0)}>1$ and, our numerical analysis confirms that in the second (final) RG stage the running value of $K_{\rho}>$ 1. Therefore the scaling dimension of the superconducting coupling is always lower than the scaling dimension of the CDW interchain coupling terms for our choice of bare values and can safely be ignored.

\section{Gapped phases}

Without disorder, an individual chain ends up in either spin-gapped (SDW or CDW) phases or a gapless Luttinger liquid phase as the temperature lowers unless the chain supports

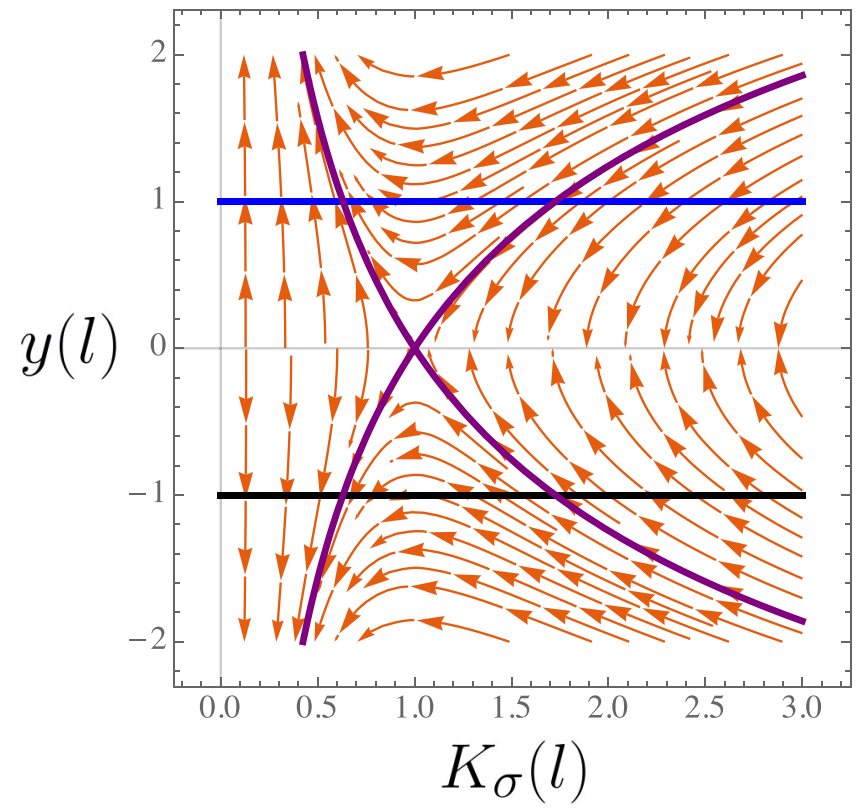

FIG. 1. The phase portrait of a system without disorder. The purple lines indicate the separatrix of the RG equations for $D=0$. The blue line indicates the SDW phase at $y=1$, whereas the black line indicates the CDW phase at $y=-1$.

an ideal spin-rotational symmetry imposing $K_{\sigma}=1$. Allowing the most generic spin anisotropic situation, the separatrix,

$$
y^{2}=8\left(K_{\sigma}^{-1}+\ln K_{\sigma}-1\right),
$$

is the phase boundary as shown in Fig. 1. When the absolute value the spin-flip interaction $y$ reaches 1, a spin gap opens leading to a SDW $(y=1)$ or CDW $(y=-1)$ phase [11]. The boundary between SDW and CDW phases is the segment of the horizontal axis $y=0,0 \leqslant K_{\sigma} \leqslant 1$. In a disordered case, solutions of the RG equations depend on the initial values (at $l=0$ ) of five parameters: $K_{\sigma}^{(0)}, y^{(0)}, K_{\rho}^{(0)}, D^{(0)}$, and $u^{(0)}$. The disorder destroys the Luttinger liquid phase for $K_{\sigma}>1$ and moves the SDW/CDW boundary $\left(K_{\sigma}<1\right)$ to the upper half-plane $y>0$. These are results of our numerical calculations of the RG equations (3)-(7) that are presented below for the typical phase diagrams for two values of $K_{\sigma}^{(0)}$ in Figs. 2 and 3. These are representative examples of the entire family of the phase diagrams parametrized by the remaining three variables. The area of different regions and the shape of boundaries may differ but the topology stays the same. We have no intention to analyze five-dimensional parametric space to present an exhaustive set of possible (but topologically equivalent) phase diagrams covering all experimental observations for microscopically distinct materials. Our task is to suggest a possible explanation of the observed in some materials enhancement of the superconducting temperature preserving features accompanying this effect. The main features, an abrupt change of the critical temperature and a linear temperature dependence of the high- $T$ resistivity, require fine-tuning. The latter imposes a constraint on the bare values of the coupling constants. The former indicates the existence of an additional phase transition in the system. The only phase transition between the conducting phases is the 


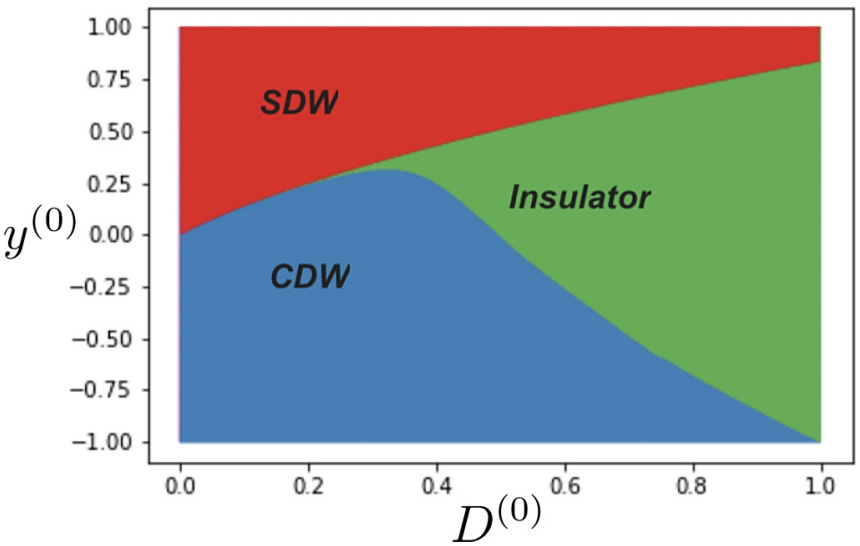

FIG. 2. $K_{\sigma}^{(0)}=0.6, K_{\rho}^{(0)}=2.2, u^{(0)}=8$ - a typical phase diagram for $K_{\sigma}<1$ displaying the SDW/CDW-boundary moved upwards by disorder and the Luttinger liquid phase destroyed.

transition between spin and charge density wave phases. We numerically scanned various RG-flows to identify a set of bare parameters where an interface exists between two conducting phases, SDW and CDW, or, at least, these two phases are separated by a numerically narrow insulating region. While Fig. 3 is encountered more frequently in simulations, Fig. 2 describes the situation that we were searching for: changing only the bare disorder strength may transfer the system between two conducting phases. In what follows, we will be focused on the situations presented by the phases diagrams with a SDW/CDW interface similar to that in Fig. 2.

In order to choose these values properly, i.e., to describe a real physical system, we address the experimental data [10]. Since we anticipate that a drastic change of the critical temperature is related to the SDW/CDW phase transition, we focus on the regions with $K_{\sigma}^{(0)}<1$ when, according to Fig. 2, we expect to have a rather sharp boundary between two spingapped phases and transition between them driven by disorder strength. This fact is also supported by the numerics taking into account multiple disorder strengths: in the Fig. 4, one can see that various disorders form two groups of trajectories and a tiny change of disorder around critical $D_{c r} \approx 0.12$ changes

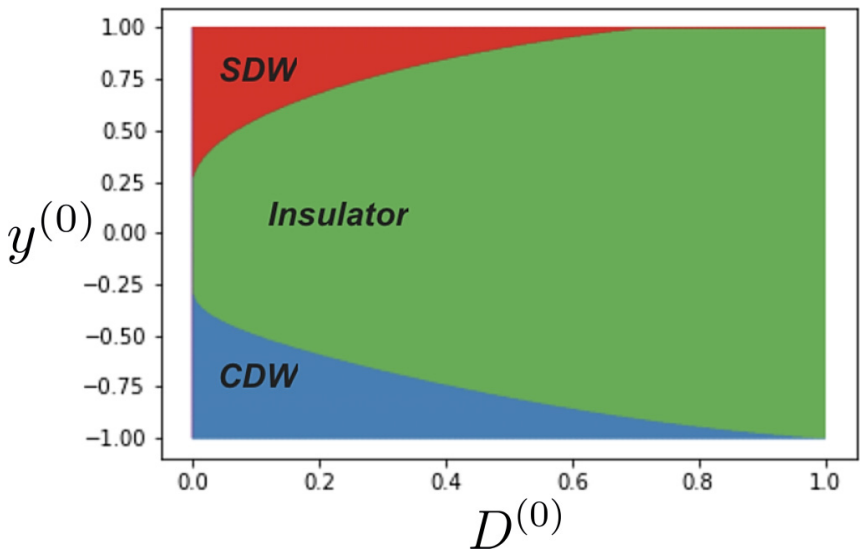

FIG. 3. $K_{\sigma}^{(0)}=1.1, K_{\rho}^{(0)}=1.5, u^{(0)}=1$ - a typical phase diagram for $K_{\sigma}>1$ with no SDW/CDW-boundary and a destroyed Luttinger liquid phase.

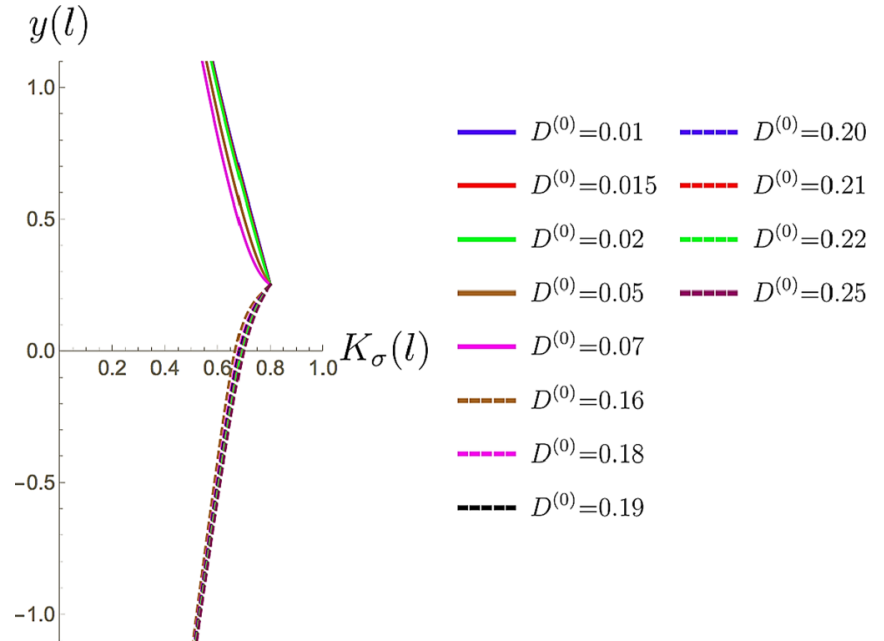

FIG. 4. The variation in $y(l)$ and $K_{\sigma}(l)$ as a function of $l$ for a disordered system. For the weak disorder, the RG trajectories flow into a SDW phase, however as disorder is increased above a critical value the system enters into a CDW phase.

drastically the end point $(y \rightarrow 1$ for SDW or $y \rightarrow-1$ for CDW).

Another requirement is that high-temperature resistivity must have a linear dependence on temperature, as observed in the experiment. In a Luttinger liquid [11], resistivity is proportional to the product of disorder and temperature $\rho \sim D(T) T$ where the (dimensionless) temperature is the running parameter of the $\mathrm{RG}$ equations, $T=e^{-l}$. The initial values of the parameters must satisfy the condition $K_{\rho}^{(0)}+K_{\sigma}^{(0)}+y^{(0)} \approx 3$ for the disorder parameter $D$ to be a constant at high temperatures. We will show below that there existence of qualitatively different RG trajectories (with increasing and decreasing $y$ ) is a necessary condition for the sharp increase of the superconducting temperature. To understand the effect of disorder on the system for a nonsuperconducting scenario, a set of initial conditions are chosen as $K_{\sigma}^{(0)}=0.8, y^{(0)}=0.25, K_{\rho}^{(0)}=1.95, u^{(0)}=8$, with $D^{(0)}=0.01,0.015,0.02,0.05,0.07,0.16,0.18,0.19,0.20$, $0.21,0.22$, and 0.25 . This range of disorders ensure that the spin gapped scenario occurs before the state becomes insulating $(D=1)$. For these set of initial conditions, RG trajectories in the $y, K_{\sigma}$-plane are presented in Fig. 4.

Please note that the phase diagrams in Figs. 2 and 3 are based on the "first step" RG equations (3)-(7) that describe how a spinful system flows into an insulating state or one of two conducting gapped phases, SDW or CDW. Further analyses (governed by the second-step RG equations) is required and it will be implemented in the following sections.

\section{SDW/CDW boundary}

Since this critical disorder represents the point at which a quantum phase transition occurs between two spin gapped phases, the characteristic length $l_{y}=-\ln T_{y}$ (at which $|y|$ reaches 1) must diverge at this critical disorder, resulting in

$$
l_{y}=\alpha\left|D^{(0)}-D_{\mathrm{cr}}^{(0)}\right|^{-v}
$$




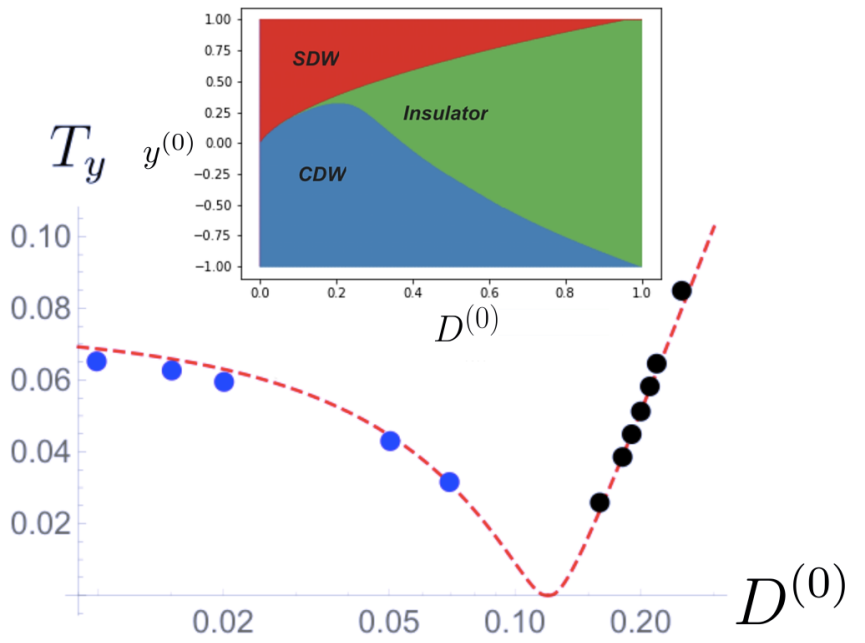

FIG. 5. The temperatures of the second-order phase transitions between the TLL and spin gapped phases as a function of the disorder for the initial conditions given by $K_{\sigma}^{(0)}=0.8, \quad y^{(0)}=0.25, \quad K_{\rho}^{(0)}=1.95, \quad u^{(0)}=8, \quad$ with $\quad D^{(0)}=$ $0.01,0.015,0.02,0.05,0.07,0.16,0.18,0.19,0.20,0.21,0.22$, and 0.25 . The red dashed line is a plot of Eq. (12), where $v=0.33$, and $\alpha=1.27$, to fit the data. For the weak (strong) disorder, blue (black) circles represent the temperature at which system goes into SDW (CDW) state. The critical disorder for the quantum phase transition is $D_{\mathrm{cr}}^{(0)} \approx 0.12$. (Inset) Phase diagram in the $y^{(0)}-D^{(0)}$ plane for $K_{\sigma}^{(0)}=0.8, K_{\rho}^{(0)}=1.95$, and $u^{(0)}=8$. This is valid for low temperatures where the TLL phase is not relevant.

where a critical exponent $v$ and a parameter $\alpha$ can be fitted according to the data. We have run simulations for a wide range of initial conditions and they resulted in the universal value $v=1 / 3$. We believe that this phase transition belongs to the mean field universality class. Indeed, we can treat spin interaction $y \sim 1 / l_{y}$ as magnetization and the deviation from the critical disorder $\left|D^{(0)}-D_{\mathrm{cr}}\right|$ as the source ("magnetic") field. At the critical temperature $T_{y}$ according to Landau phase transition theory the source field is proportional to the magnetization in the power $\delta=3$, which immediately leads to $v=1 / \delta=1 / 3$. Numerical results and analytical dependence of the temperature $T_{y}$ on the initial disorder $D^{(0)}$ for the initial conditions described above and presented in Fig. 5 are in almost perfect agreement.

In the inset of Fig. 5, we show the phase diagram for the initial values used above. It shows that depending on the initial point the system enters into one of three possible phases: SDW, CDW or insulating. The temperature dependence presented in Fig. 5 corresponds to a line $\left(y^{(0)}=0.25\right)$ in the inset which passes through a very narrow region of insulating phases, which allowed us to fit both branches in Fig. 5 with the same value of the critical disorder.

\section{E. High-temperature resistivity}

When the interaction strength $y$ between opposite spins reaches an absolute value of unity, a system enters in one of two spin-gapped phases and therefore the spin degrees of freedom freeze. As we have mentioned above, this work was inspired by experimental results [10] where high- $T$ resistivity

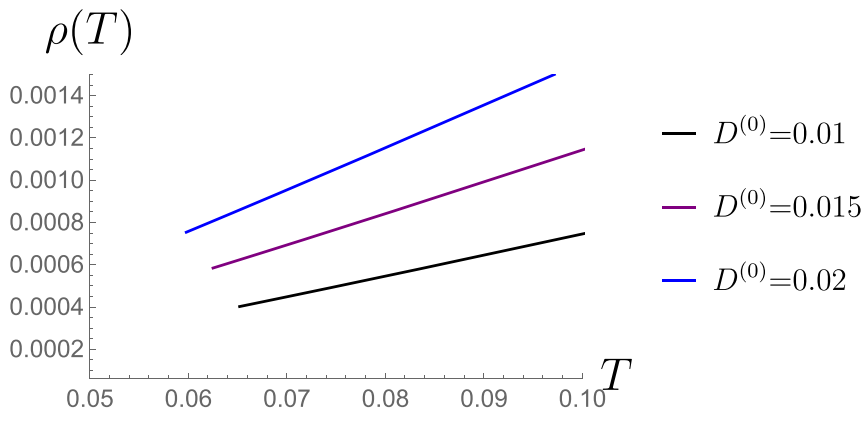

FIG. 6. High- $T$ resistivity for different disorders. The graphs are cut at the minimum of the resistivity where the spin gap opens at lower temperatures because the system goes into a SDW phase. The resisitivity would continue upwards for the spin gapped set of RG equations (8) and (9).

demonstrated linear in $T$ dependence at high temperatures. Since RG analysis can be formulated as dimensionless temperature (running cutoff) $T=e^{-l}$ dependence of the disorder strength, the resistivity behaves like $\rho(T) \sim T D(T)$ [11]. To reproduce linear- $T$ resistivity, we will always be choosing the bare values of parameters such that the initial scaling dimension of disorder is close to the dimension of space + time, i.e., satisfies the condition $K_{\rho}^{(0)}+K_{\sigma}^{(0)}+y^{(0)} \approx 3$ to match the experimental graphs. We present two different situations with linear dependence of resistivity found from equations (3-7). For a weak disorder, the system goes into SDW phase after the linear decrease with lowering temperature, Fig. 6. For stronger initial disorders, the resistivity minimum is always reached at temperatures $T_{\min }>T_{y-}$ which is shown in Fig. 7 where a CDW spin gap $y=-1$ (black circles in Fig. 5) can only open after the minimum. When superconductivity is included, this may also occur before the CDW phase is reached. It is important to stress that without superconductivity, the state will enter a gapped phase or an insulator as temperature

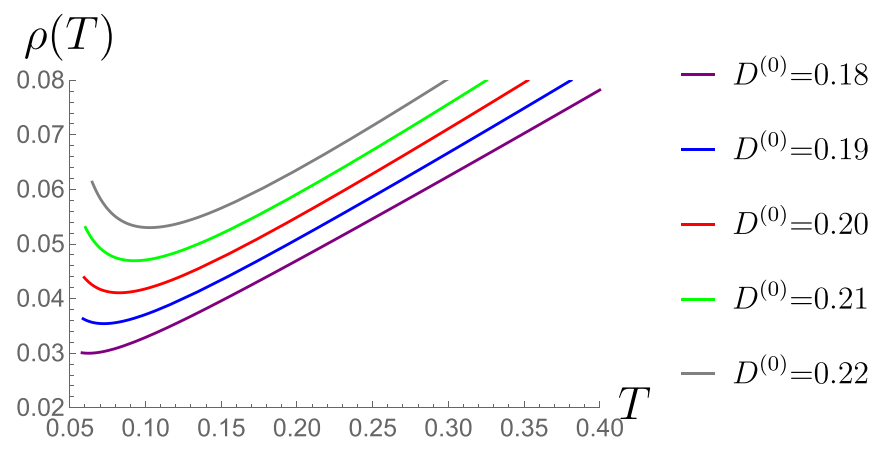

FIG. 7. This figure shows the minimum in resistivity occurs before any spin gapped or superconducting phase is reached. When the lines on the figure after the upturn stop, this indicates that the system has entered either a superconducting phase or a CDW phase. The only disorder which enters a CDW phase is the strongest disorder $\left(D^{(0)}=0.22\right)$ as superconductivity emerges before the spin gapped phase for the lower disorders. For the CDW phase, the spin gapped RG equations must be solved, and the value at which superconductivity occurs must be found. The onset of superconductivity is discussed in more detail in the next section. 


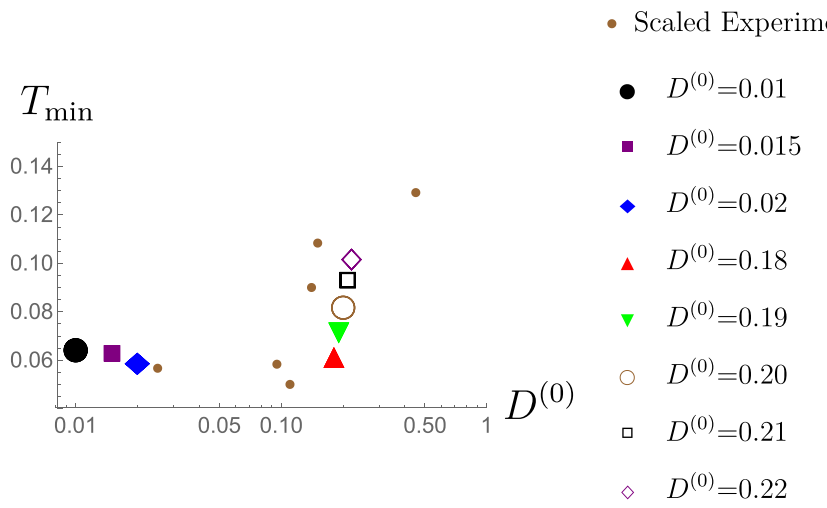

FIG. 8. Resistivity minimum temperature as function of disorder. Three left symbols correspond to $y=+1$, small brown circles are scaled experimental results, and five right symbols correspond to a "real" resistivity minimum.

is lowered. This was the main result of the previous section. The initial conditions when including superconductivity are discussed in the next section. In order to reproduce the experimental results, we scale them and draw against our numerics in Fig. 8. The high temperature resistivity in the experiment [10] was the measure of the disorder, we scale it as $10^{5}$ and divide experimental temperatures by 1200 . The experimental results with our numerical values $T_{y+}$ and $T_{\min }$ are presented in Fig. 8. Even though we cannot reproduce a kink observed in the experiment, we nevertheless do see a good quantitative agreement in the sharp increase of the temperature near the critical disorder. Our numerical simulations show that the curves for $T_{\min }$ and $T_{y-}$ are almost parallel, and therefore the relation between $T_{y+}$ and $T_{\min }$ resembles the relation between two branches $T_{y+}$ and $T_{y-}$ of the phase transiton in Fig. 2. The fact that $T_{\min }>T_{y-}$ simply means that $T_{y+}$ and $T_{\min }$ have different parameteres $\alpha$ in scaling $\exp \left[-\alpha\left|D^{(0)}-D_{c r}\right|^{-v}\right]$, whereas $T_{y+}$ and $T_{y-}$ have the same parameter $\alpha$ in Fig. 5.

This temperature $T_{y+}$ (blue circles in Fig. 5) defines the minimum of the resistivity for the following reasons. When a spin gap opens, two closely related effects take place: the gap freezes the spin density field at such a value that the average backscattering term due to disorder goes to zero, decreasing resistivity, and both spin parameters $y$ and $K_{\sigma}$ do not contribute to the scaling dimensions, and resistivity $D T$ (which now has a scaling dimension $K_{\rho}<2$ ) immediately starts to grow with decreasing temperature.

\section{SUPERCONDUCTIVITY}

The transition to a superconducting state is always preceded by an insulating behavior resulting in a peak of resistivity with following an immediate drop to zero. We cannot describe the exact shape of this curve and find the critical temperature. Instead we assume that the drop is caused by the proximity of a superconducting instability and it happens at $T_{\text {peak }}$ when the effective dimensionless Josephson coupling becomes of the order of one, $J\left(T_{\text {peak }}\right)=1$. We have chosen the initial value of the Josephson coupling $J^{(0)}=0.115$ to present the appearance of the superconductivity on the graph, Fig. 9. The graph of $T_{\text {peak }}$ in Fig. 9 exhibits disorder-induced

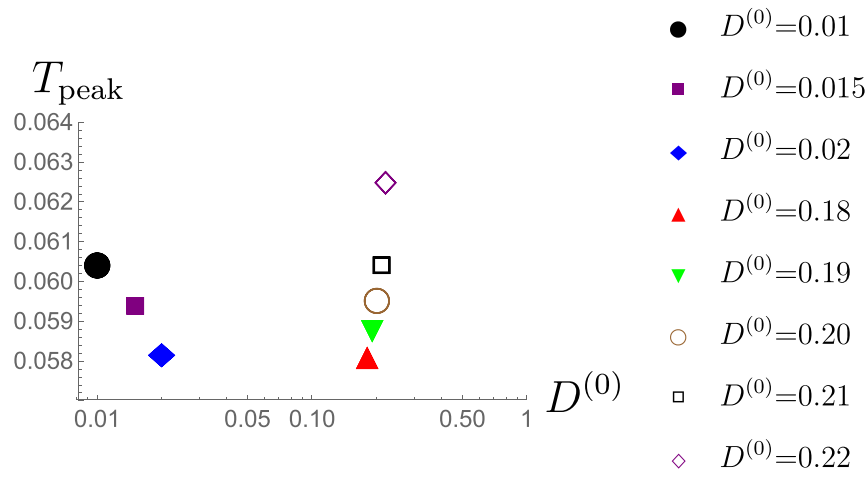

FIG. 9. $T_{\text {peak }}$ as function of disorder, including the spin gapped mechanisms. This figure shows a plateau from the third weakest disorder to the next strongest disorder. The three weakest disorders occurs via a SDW mechanism. There is an almost linear increase in $T_{\text {peak }}$ as disorder increases from the gapless phases through to the CDW phase. The strongest disorder occurs via a CDW mechanism, which has a larger relative increase. This does suggest a CDW mechanism is favourable for disorder-induced superconductivity.

superconductivity, and there is a clear plateau from the third weakest disorder to the next strongest disorder. However, the second plateau that was expected is not revealed, as the CDW mechanism seems to enhance disorder-induced superconductivity.

It is worth drawing attention to the fact that were we solving the problem of the superconducting transition assuming that the chains are in a spin-gapped phase at all temperatures (i.e. even at the bare values of later renormalized parameters), it would be sufficient to analyze the second-step RG equations (8)-(10) only. This approach has been previously developed [15], however the main drawback was that they did not care to separate genuine inelastic processes from the elastic ones caused by disorder and that inaccuracy led to a wrong RG equation (8), and as the result, to an incorrect dependence of the critical temperature on the disorder strength. Even if this proposal used the correct RG equations, their results would demonstrate a suppression of the critical temperature for all but abnormally large values of $K_{\rho}^{(0)}$.

The result of this paper is the enhancement of the critical temperature in some region of the bare parameters which is entirely governed by the RG flow in the gapless phase. This first-step RG flow turns out to be extremely sensitive to the disorder strength when the bare parameters are chosen close to the separatrix of a clean Luttinger liquid. The slight change of disorder causes a drastic consequence - which gapped state, SDW or CDW, the chain enters depends on the disorder strength. Subsequently, in which gapped phase the superconducting order starts to develop depends on the disorder. Since CDW is favourable for superconducting correlations (in comparison to the SDW phase) and disorder increase leads to the CDW phase, we may conclude that the disorder indirectly enhances superconductivity.

Another qualitative explanation of the different dependence of the superconductivity on the disorder in different spin-gapped phases is based on the fact that the superconducting parameter $J$ is related to a singlet interchannel coupling. The combination of singlet intrachannel and interchannel 
couplings (in the CDW phase) results in a higher superconducting temperature than the combination of triplet intrachannel and singlet interchannel couplings (in the SDW phase). The fact that the disorder strength drives the quasi- $1 D$ system from a SDW to CDW phase leads to the disorderenhanced superconductivity phenomenon.

\section{CONCLUSIONS}

We have studied disorder-induced and enhanced superconductivity in a quasi- $1 D$ correlated system using RG equations. Our results show that for a wide range of bare parameters, the system undergoes a second-order disorder-induced phase transition between the SDW phase (for the three smallest disorders), a gapless phase (the next four disorders), and the CDW phase (for the strongest disorder). The opening of a spin gap in collaboration with an interchain singlet Josephson coupling leads to qualitatively different dependencies of critical superconducting temperature: a weak decrease of the critical temperature with an increase of the disorder while in the SDW phase, and an almost linear increase of the critical temperature with an increase of the disorder from the gapless to the CDW phase, which becomes truncated at stronger disorder because the system eventually is driven into an insulating phase at very strong disorder. The analysis shows that the tendency from SDW to CDW via the gapless phase provides a favourable scenario for superconductivity to occur. This behavior is driven by disorder and proves that imperfection may enhance superconductivity and even induce it. Our results are in good agreement with the outstanding experimental results [10] for both, temperatures of the resistivity minima (quantitatively), and superconductivity transition temperatures (qualitatively).

\section{ACKNOWLEDGMENTS}

This work was supported by the Leverhulme Trust Grants No. RPG-2016-044 (IY) and No. RPG-2019-317 (AL, IY), and by MOST/MESU Grant No. 3-16430 (VK). IVY would like to thank Prof. V. Kravtsov for drawing our attention to the paper [10] and the problem in general. The authors (VK and IVY) are grateful for hospitality extended to them at the Center for Theoretical Physics of Complex Systems, Daejeon, South Korea.

\section{APPENDIX: RENORMALIZATION GROUP ANALYSIS}

We are using the RG equations first derived in Ref. [12]:

$$
\begin{aligned}
\frac{d \tilde{y}}{d l} & =-2\left(\tilde{K}_{\sigma}-1\right) \tilde{y}-D, \\
\frac{d \tilde{K}_{\sigma}}{d l} & =-\frac{1}{2} \tilde{K}_{\sigma}^{2} \tilde{y}^{2}-\frac{1}{2} \tilde{K}_{\sigma}^{2} D, \\
\frac{d \tilde{K}_{\rho}}{d l} & =-\frac{1}{2} \tilde{K}_{\rho}^{2} \frac{u_{\rho}}{u_{\sigma}} D, \\
\frac{d D}{d l} & =-\left(\tilde{K}_{\sigma}+\tilde{K}_{\rho}+\tilde{y}-3\right) D, \\
\frac{d \tilde{u}_{\rho}}{d l} & =-\frac{\tilde{u}_{\rho}^{2}}{2 \tilde{u}_{\sigma}} \tilde{K}_{\rho} D,
\end{aligned}
$$

$$
\frac{d \tilde{u}_{\sigma}}{d l}=-\frac{\tilde{u}_{\sigma} \tilde{K}_{\sigma}}{2} D
$$

The parameters are tilded since a running cutoff was introduced when evaluating a nonlocal in time disorder term in the action. Such a procedure introduces a spurious inelastic interaction and creates an admixture of the interaction and disorder. It was first realized in [12] that this subtlety must be healed by "disentanglement" of disorder and interaction contributions to the RG by redefinition of the parameters. This procedure was first applied in [12] to the weak interaction approximation and further explained in Ref. [11]. We follow a more general approach developed in [13] to deal with arbitrary interaction strength. In terms of standard $g$-ology [11], the strength of the interaction between opposite spins $g_{1 \perp}$ requires a redefinition:

$$
\tilde{g}_{1 \perp}(l)=g_{1 \perp}(l)-\frac{2 D_{b} \alpha}{u_{\sigma}} .
$$

To incorporate equation (A7) into the other parameters, an expansion for small disorder can be performed, which is consistent with the limitation of the RG theory which is perturbative in weak disorder parameter $D$ :

$$
\begin{aligned}
\tilde{K}_{\rho} & =K_{\rho}-\frac{K_{\rho}^{2}+1}{4} D \gamma, \\
\tilde{K}_{\sigma} & =K_{\sigma}-\frac{K_{\sigma}^{2}+1}{4} D \gamma, \\
\tilde{y} & =y-D \gamma, \\
\tilde{u}_{\sigma} & =u_{\sigma}+\frac{K_{\sigma}^{2}-1}{4 K_{\sigma}} u_{\sigma} D \gamma, \\
\tilde{u}_{\rho} & =u_{\rho}+\frac{K_{\rho}^{2}-1}{4 K_{\rho}} u_{\rho} D \gamma,
\end{aligned}
$$

where

$$
\gamma=\left(\frac{u_{\rho}^{(0)} K_{\rho}}{K_{\rho}^{(0)} u_{\sigma}}\right)^{K_{\rho}} .
$$

Before converting equations (A1)-(A6) back into the bare parameters, it can be seen that the ratio between $\tilde{u}_{\rho} / \tilde{K}_{\rho}$ does not renormalize. This allows the removal of $\tilde{u}_{\rho}$ as a varying parameter by substituting

$$
\tilde{u}_{\rho}=\left(\frac{\tilde{u}_{\rho}}{\tilde{K}_{\rho}}\right) \tilde{K}_{\rho}=\left(\frac{\tilde{u}_{\rho}^{(0)}}{\tilde{K}_{\rho}^{(0)}}\right) \tilde{K}_{\rho},
$$

where $(0)$ denotes the parameters at $l=0$, i.e., bare value of the parameters. By doing this, the system goes from seven varying parameters to six. This obviously reduces the complexity of the system, and allows for better analytical tractability. By now using both sets of equations (A1)-(A6) and (A8)-(A12), the complete set of correct RG equations can be written in terms of their real parameters as equations (3) (7).

Now the model is written in terms of their bare parameters in a set of coupled first order differential equations. The challenge remains in determining a set of initial conditions which exhibit disorder-induced superconductivity. The scaling dimension of the Josephson coupling term is known [11] 
and the RG equation is easily written:

$$
\frac{d J}{d l}=\left(2-K_{\sigma}-\frac{1}{K_{\rho}}\right) J .
$$

Since there is no back action from $J$ to the renormalization of other parameters, it is sufficient to solve those RG equations and then restore renormalization of the effective Josephson coupling,

$$
J(l)=J^{(0)} \exp \left\{\int_{0}^{l} d l^{\prime}\left[2-K_{\sigma}-K_{\rho}^{-1}\right]\right\},
$$

with a stop at some critical $l_{c}$ when $J\left(l_{c}\right)=\Lambda_{0}$. The transition temperature is then found as $T_{c}=\Lambda_{0} e^{-l_{c}}$. In the main text, we are using dimensionless parameters which implies that all energies are normalized by the upper cutoff $\Lambda_{0}$. In this scenario, there are many sets of initial conditions that could be implemented. To determine what would be an appropriate set of conditions, we may notice that starting around a separatrix [11],

$$
y^{2}=8\left(K_{\sigma}^{-1}+\ln K_{\sigma}-1\right) .
$$

which is a boundary between different phases of a clean system, even small disorder is expected to make a dramatic change and drastically modify the RG trajectory forcing a disorder-induced phase transition in the system. By choosing initial values (bare values of the parameters) in the vicinity of the separatrix, the belief is that the system undergoes the phase transition and superconductivity will be developing accordingly.
[1] P. W. Anderson, Theory of dirty superconductors, J. Phys. Chem. Solids 11, 26 (1959).

[2] P. W. Anderson, Absence of diffusion in certain random lattices, Phys. Rev. 109, 1492 (1958).

[3] M. Ma and P. A. Lee, Localized superconductors, Phys. Rev. B 32, 5658 (1985).

[4] A. M. Finkelstein, Superconducting transition temperature in amorphous films, JETP Lett. 45, 46 (1987).

[5] Y. Oreg and A. M. Finkel'stein, Suppression of $T_{c}$ in Superconducting Amorphous Wires, Phys. Rev. Lett. 83, 191 (1999).

[6] I. V. Yurkevich and I. V. Lerner, Nonlinear sigma model for disordered superconductors, Phys. Rev. B 63, 064522 (2001).

[7] M. V. Feigel'man, L. B. Ioffe, V. E. Kravtsov, and E. Cuevas, Fractal superconductivity near localization threshold, Ann. Phys. 325, 1390 (2010).

[8] I. S. Burmistrov, I. V. Gornyi, and A. D. Mirlin, Enhancement of the Critical Temperature of Superconductors by Anderson Localization, Phys. Rev. Lett. 108, 017002 (2012).

[9] E. J. König, A. Levchenko, I. V. Protopopov, I. V. Gornyi, I. S. Burmistrov, and A. D. Mirlin, Berezinskii-kosterlitz-thouless transition in homogeneously disordered superconducting films, Phys. Rev. B 92, 214503 (2015).

[10] A. P. Petrović, D. Ansermet, D. Chernyshov, M. Hoesch, D. Salloum, P. Gougeon, M. Potel, L. Boeri, and C. Panagopoulos, A disorder-enhanced quasi-one-dimensional superconductor, Nat. Commun. 7, 12262 (2016).

[11] T. Giamarchi, Quantum Physics in One Dimension (Clarendon, Oxford, 2004).

[12] T. Giamarchi and H. J. Schulz, Anderson localization and interactions in one-dimensional metals, Phys. Rev. B 37, 325 (1988).

[13] I. V. Gornyi, A. D. Mirlin, and D. G. Polyakov, Electron transport in a disordered luttinger liquid, Phys. Rev. B 75, 085421 (2007).

[14] S. T. Carr and A. M. Tsvelik, Superconductivity and chargedensity waves in a quasi-one-dimensional spin-gap system, Phys. Rev. B 65, 195121 (2002).

[15] Y. Suzumura and T. Giamarchi, Impurity Pinning vs Superconductivity in Quasi-One-Dimensional Electron Systems, J. Phys. Soc. Jpn. 58, 1748 (1989). 